\title{
Ferienzeit ist Reisezeit
}

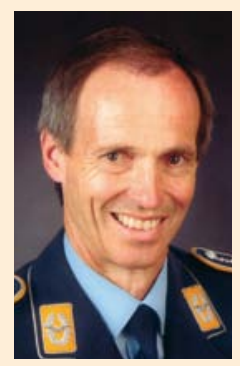

Liebe Kolleginnen und Kollegen,

die Reiselust der Menschen ist ungebrochen und dies kommt glücklicherweise auch dem Tourismus in Deutschland zugute. Andererseits stabilisieren sich Fernreisen auf einem hohen Niveau. Beratung und Betreuung bleiben auf diesem Gebiet daher wichtig und die dementsprechenden Themen sind durch uns immer wieder und erneut aufzugreifen, so auch in diesem Heft.

Als Kasuistik wird der Fall einer AV-Knoten-Reentrytachykardie (AVNRT) bei einem Verkehrsflugzeugführer vorgestellt, der erfolgreich mit einer Katheterablation mittels Hochfrequenztechnik behandelt wurde. Dr. Martin Trammer von der DLR in Köln, Prof. Dr. Marc Horlitz von der Kardiologischen Klinik des Krankenhauses in Porz et al. zeigen, dass mit der dabei verwandten modernen Technik bei circa 20000 Eingriffen im Jahr primäre Erfolgsraten von 90-98\% erzielt werden können. Die Gefahr eines AV-Blockes 3. Grades liegt bei unter 1\%, in circa $5 \%$ der Fälle muss bis zu 8 Wochen nach dem Eingriff mit einem Rezidiv gerechnet werden. In den meisten Fällen kann spätestens nach Ablauf eines Jahres uneingeschränkte Tauglichkeit testiert werden.

Der Gesundheit von Seeleuten widmet sich eine Pilotstudie von Swaantje Klostermann, MPH, aus München und Dr. Thomas Claßen aus Bielefeld. Im Vordergrund stand die subjektive Einschätzung der Seeleute zu ihrer Gesundheit und zur Gesundheitsförderug sowie deren Bedarf. Die Befragung erfolgte schriftlich, die Bereitschaft zur Teilnahme war groß. Eine medizinische Versorgung an Bord hielten alle für wichtig oder sehr wichtig, 90\% wünschen für die Zukunft Gesundheitsprogramme an Bord und an Land. Der Gesundheitszustand der Seeleute ist nicht schlechter als der der Allgemeinbevölkerung.

Die Problematik von Vergiftungen nach Schlangenbiss ruft uns Prof. Dr. Dietrich Mebs vom Institut für Rechtsmedizin in Frankfurt in Erinnerung. Er zeigt auf, dass die Symptome vielfältig sein können und von der Spezies der Schlange abhängen. Die einzige spezifische Therapie sind Antidote als Antiseren. Eine ärztliche Hilfe vor Ort ist notwendig, da Antiseren in der Kühlkette gehalten werden müssen und dementsprechend nicht als Reiseapotheke mitgeführt werden können. Manipulationen an der Bissstelle sind zu unterlassen. Wegen der Gefahr der Anaphylaxie bei der notwendigen intravenösen Anwendung der Antiseren ist eine klinische Überwachung empfehlenswert. Obwohl jährlich mehr als 125000 Menschen infolge von Schlangenbissen sterben, ist die Gefahr für Touristen eher gering, zumal wenn diese gängige Vorsichtsmaßnahmen beherzigen. Sollte kein Antiserum zur Verfügung stehen, muss symptomatisch behandelt und gegebenenfalls zu Hause nachbehandelt werden.

Eine Pilotstudie in einer hausärztlichen Gemeinschaftspraxis beschäftigt sich mit dem Impfstatus der Patienten. Nora Stigrot, Sibylla Krane und Dr. Jörg Schelling aus München und Martinsried zeigen, dass die Impfquoten in einer Praxis verbessert werden können, wenn eine EDV-gestützte Impfplanung und -überwachung in das Praxisverwaltungssystem integriert ist. Eine umfassende und hochwertige Aufklärung und Beratung ist jedoch unverzichtbar.

Louise Reher und Dr. Jakob Cramer aus Hamburg geben eine umfassende Übersicht über die Notwendigkeit der Malariaprophylaxe und die Notfalltherapie in der Schwangerschaft. Bei über 100 Mio. Reisenden in Malariagebiete ist eine Malariainfektion in der Schwangerschaft ein ernst zu nehmendes und nicht seltenes Risiko. Schwangere Frauen sollten auf solche Reisen verzichten, da Erkrankungsfolgen schwerwiegend sein können. Entscheidend ist die Expositionsprophylaxe, für Schwangere Mefloquin als medikamentöse Prophylaxe und Notfalltherapie empfohlen. 